\title{
A design procedure for evaluation and prediction of in-situ cemented backfill performance
}

\author{
Xiaoming Wei \& Lijie Guo \\ National Centre for International Research on Green Metal Mining, BGRIMM Technology Group, Beijing, \\ China
}

\begin{abstract}
To dispose of the smelting slag and reduce backfill cost in the mine, the aggregate mixed with smelting slag was selected as the filling aggregate, and then the filling industrial test of smelting slag was carried out in the stopes. Through the geological coring and strength test, the in-situ cemented backfill mass had been evaluated by the comparative analysis of RQD、P-wave velocity and UCS, which proved that the gradation of filling aggregate was optimized, and the quality of in-situ cemented backfill was improved by adding smelting slag. Based on internal links of physicomechanical parameters of cemented backfill, a relation between UCS and P-wave modulus was established from the perspective of dimensional balance. According to the test data of dry density, P-wave velocity and UCS of in-situ cemented backfill, the UCS prediction formula was obtained by the linear fitting method, which provided a strong research basis for the comprehensive quality evaluation of subsequent backfill.
\end{abstract}

Keywords: mixed aggregates, smelting slag, in-situ coring, quality of cemented backfill, prediction formula

\section{INTRODUCTION}

The Kalatongke Copper-Nickel mine was located in Fuyun County, Xinjiang. It was a nonferrous metal company integrating mining, mineral processing and smelting (Bing and Jun, 2003; Xiaosu, 2009). At present, Gobi aggregate were used as filling aggregate in the mine (Chen et al., 2018). With the continuous improvement of national environmental protection requirements in recent years, procurement costs had risen sharply, and a large amount of smelting slag in the mine was also facing high disposal costs. In order to reduce the filling cost, the filling aggregate optimization test was carried out . Through in-situ coring and strength testing of cemented backfill in the underground industrial stope, the quality of in-situ backfill was systematically evaluated to guarantee the safety of stope (Ghirian and Fall, 2013; Di and Sijing, 2015).

\section{TEST MATERIALS}

The gobi aggregate and smelting slag were used as filling aggregates in the test stope, which the content of smelting slag was $12 \%$ of the total mass of aggregates. The cementitious material was cement, and the slurry concentration was $84 \%$. The physical and mechanical parameters of Gobi aggregate and smelting slag were shown in Table 1. The particle size distribution were shown in Figure 1. 
Table 1. The physical and mechanical parameters of Gobi aggregate and smelting slag.

\begin{tabular}{llll}
\hline \multicolumn{1}{c}{ Aggregate } & Density $/\left(\mathrm{g} \cdot \mathrm{cm}^{-3}\right)$ & Bulk density $/\left(\mathrm{g} \cdot \mathrm{cm}^{-3}\right)$ & Porosity \\
\hline Gobi aggregate & 2.50 & 1.36 & 0.46 \\
Smelting slag & 3.57 & 1.99 & 0.44 \\
\hline
\end{tabular}

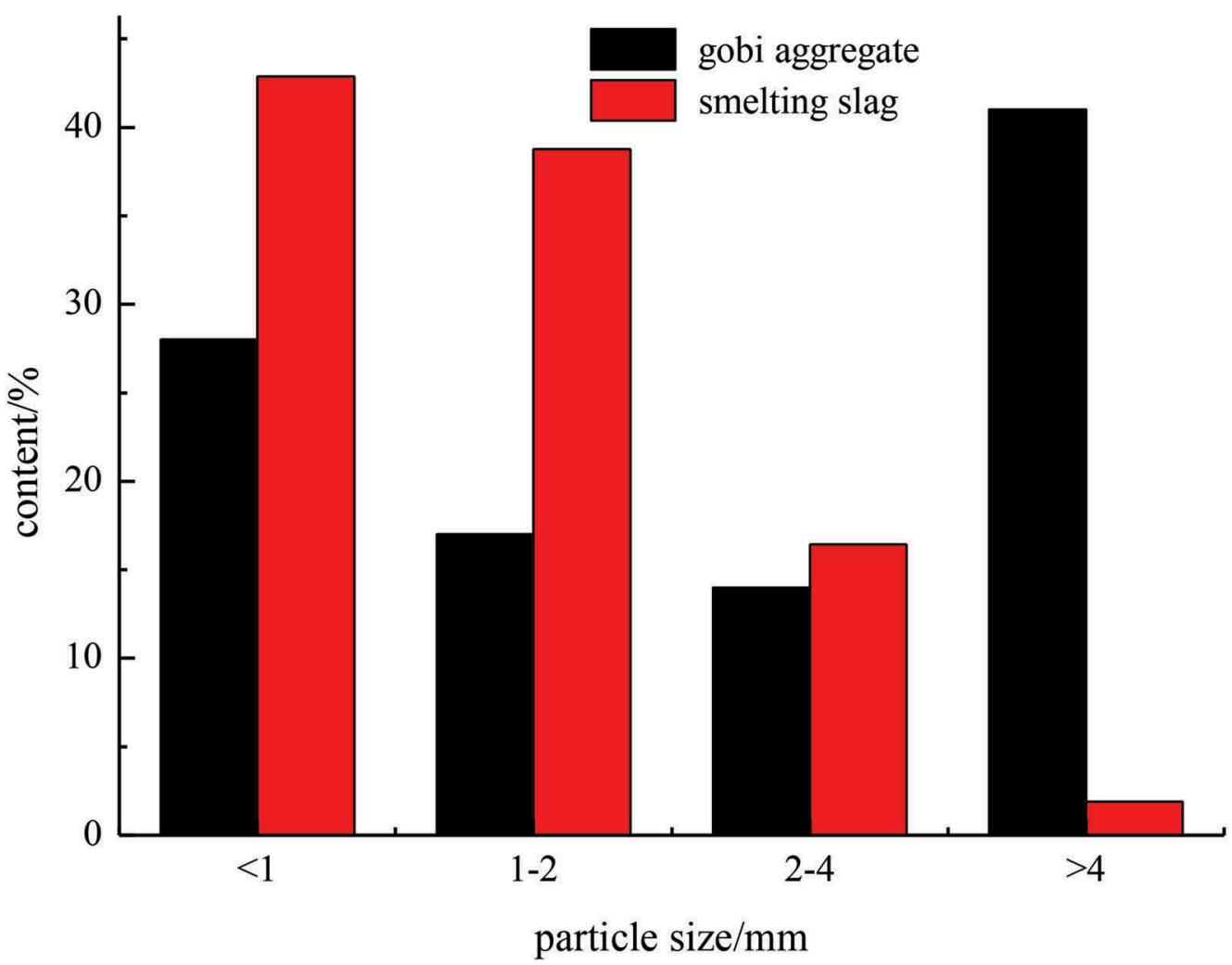

Figure 1. The particle size distribution.

\section{FILLING INDUSTRIAL TEST}

\subsection{Selection of test stope}

At present, the downward layered cemented filling method was adopted by the mine. The strength requirement of the bottom layer of backfill was greater than $3 \mathrm{MPa}$. Therefore, the E3 route was selected as the smelting slag test stope, S4 route was used as comparative test stope without smelting slag. The 1:5 of cement-sand ratio was designed in filling bottom layer, and the filling height is $2 \mathrm{~m}$.

\subsection{Filling process}

According to the existing conditions of the 1\# filling station, the gravel silo was used as the smelting slag silo, and the discharge port was modified to realize the stable transportation of the smelting slag. Through the flow calibration test, a regulating valve was set at the discharge 


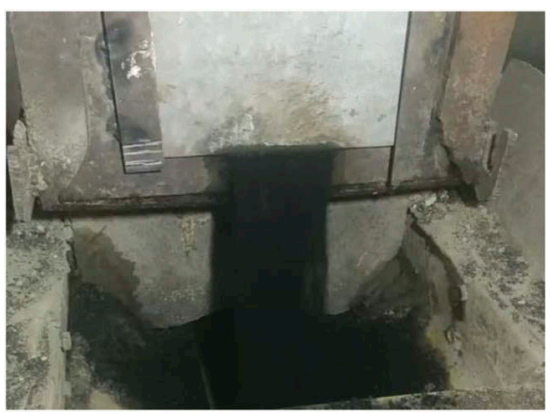

Figure 2. The regulating valve.

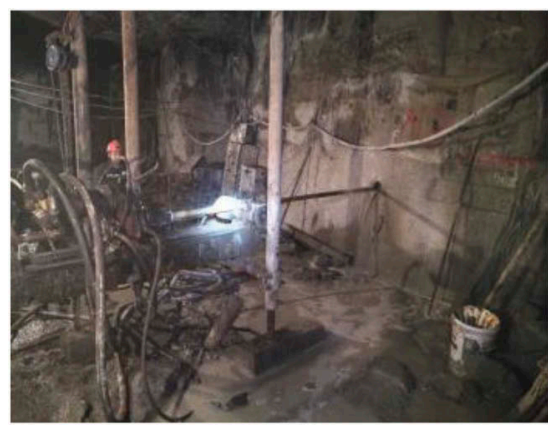

Figure 3. The coring arrangement.

port, as shown in Figure 2. The smelting slag and Gobi aggregate were sent to the mixing tank. After being stirred, the filling slurry was transported to the stope.

\subsection{Sampling of test stopes}

In the filling process, the casting tests were performed in each stope. When the test blocks was placed in a underground stope for curing 60 days, then the uniaxial compressive strength was determined. After the cemented backfill of two test stopes were cured for 60 days, in the $926 \mathrm{~m}$ level a geological drill was used for coring detection, and the coring arrangement was shown in Figure 3. Meanwhile the return data and the integrity of the coring samples were recorded. Based on the RQD, P-wave velocity and uniaxial compressive strength (UCS), the quality of in-situ cemented backfill was evaluated .

\section{QUALITY EVALUATION OF IN-SITU CEMENTED BACKFILL}

\subsection{RQD}

The coring length of bottom layer at S4 route was $20 \mathrm{~m}$, there were 3 sets of samples, as shown in Figure 4. A total of 67 standard samples were processed. From the perspective of sensory quality, the $0 \sim 11.5 \mathrm{~m}$ of coring samples were basically complete, even thickness, local fragmentations. After $11.5 \mathrm{~m}$, the coring samples was relatively broken. The RQD of S4 route was $60 \%$. The coring length of bottom layer at E3 route was $26.5 \mathrm{~m}$, there were 5 sets of samples, as shown in Figure 4. A total of 120 standard samples were processed. From the perspective of sensory quality, the coring samples were complete, smooth surface, even thickness. The RQD of E3 route was $85 \%$.

Through the comparative analysis of the RQD of the two test stopes, the RQD of E3 route was higher than S4 route, which proved that the integrity of the coring cemented backfill of the $\mathrm{E} 3$ route was better than $\mathrm{S} 4$ route.

\subsection{P-wave velocity}

The P-wave velocity distribution of S4 and E3 routes were shown in Figure 5. The P-wave velocity distribution of the $\mathrm{S} 4$ coring samples was discrete, and the average wave velocity was $2515 \mathrm{~m} / \mathrm{s}$. The P-wave velocity distribution of the E3 coring samples was concentrated, and the average wave velocity was $2649 \mathrm{~m} / \mathrm{s}$. Through the comparative analysis of the P-wave velocity of the two test stopes, the average wave velocity of E3 route was higher than S4 route, which proved that the compacting and homogeneity of the coring cemented backfill of the E3 route was better than $\mathrm{S} 4$ route. 


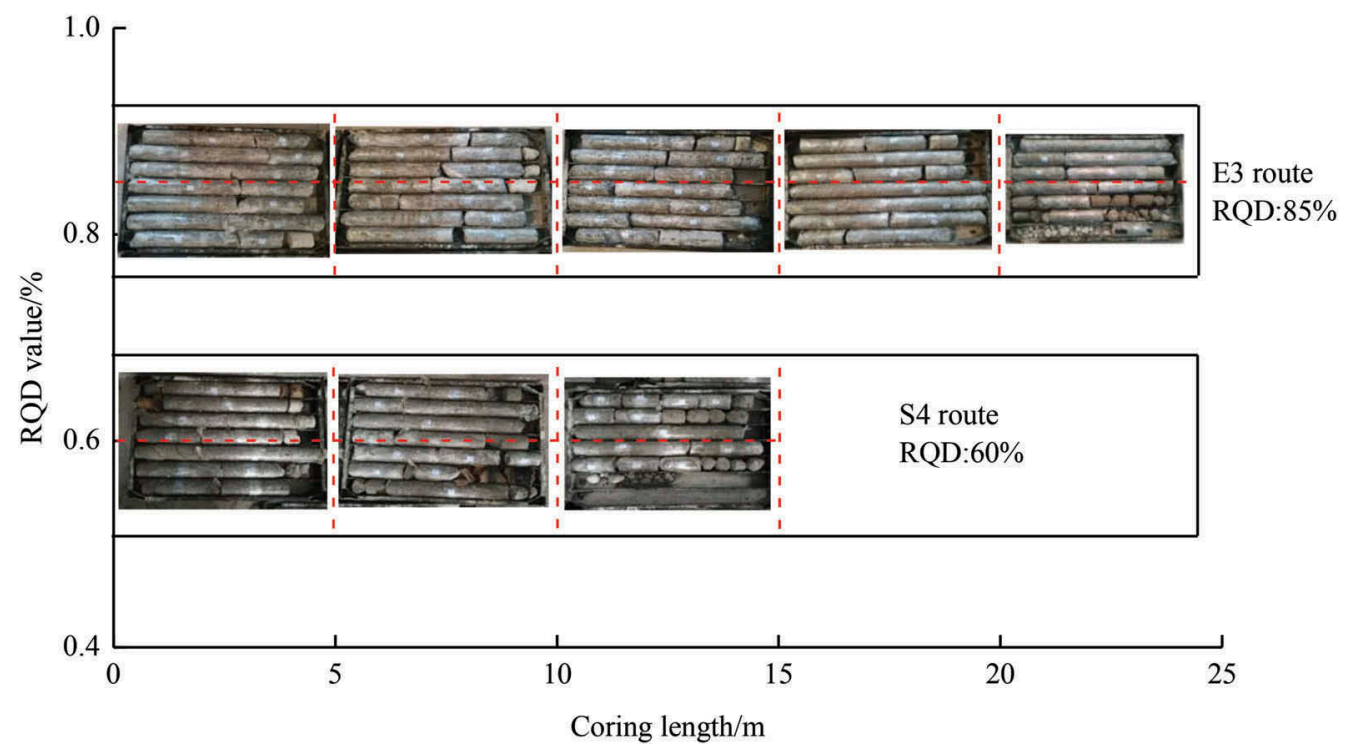

Figure 4. In-situ cemented backfill samples of S4 and E3 routes.

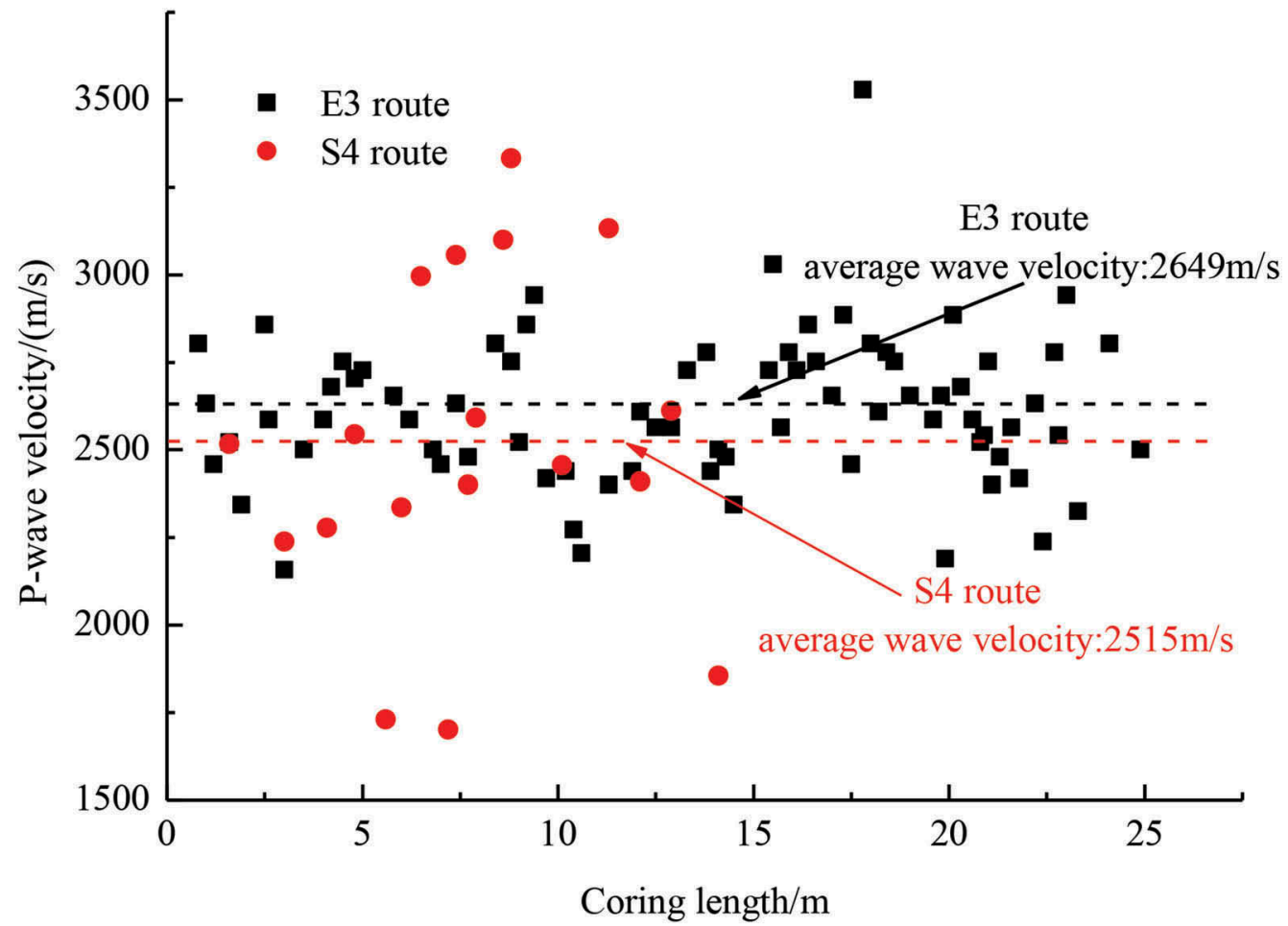

Figure 5. The P-wave velocity distribution of S4 and E3 routes. 


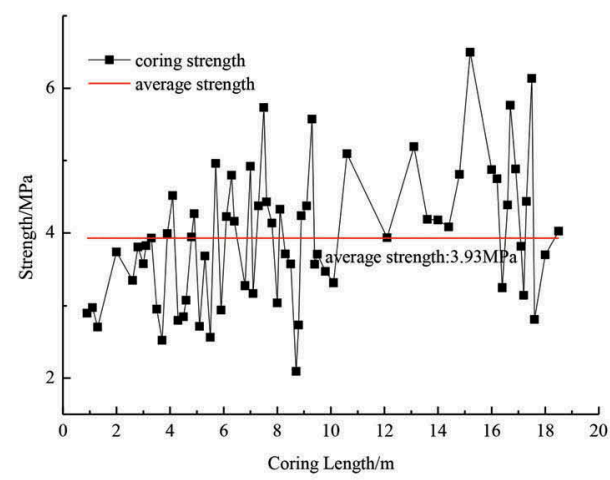

(a) $\mathrm{S} 4$ route

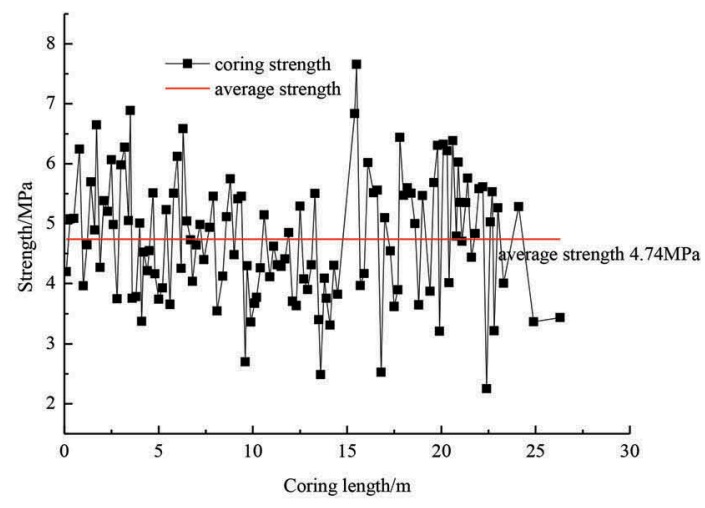

(b) E3 route

Figure 6. The UCS distribution of S4 and E3 routes.

\section{$4.3 \quad U C S$}

The UCS distribution of S4 and E3 routes were shown in Figure 6. The average strength of the S4 and E3 coring samples were 3.93MPa and 4.74MPa respectively. The average strength of the curing blocks were $4.1 \mathrm{MPa}$ and $5.2 \mathrm{MPa}$ respectively. Through the comparative analysis of the UCS of the two test stopes, the average strength of E3 route was higher than S4 route, which proved that the consolidation of the coring cemented backfill of the E3 route was better than $\mathrm{S} 4$ route.

\section{UCS PREDICTION FORMULA OF IN-SITU CEMENTED BACKFILL}

\subsection{Theoretical derivation}

In the elastic theory (Weiguo and Yulong, 2007; Moose et al., 2001), the three-dimensional wave equation in uniform, isotropic, and ideal elastic medium was

$$
\left.\begin{array}{l}
(\lambda+\mu) \frac{\partial \theta}{\partial x}+\mu \nabla^{2} u-\rho \frac{\partial^{2} u}{\partial t^{2}}=0 \\
(\lambda+\mu) \frac{\partial \theta}{\partial y}+\mu \nabla^{2} v-\rho \frac{\partial^{2} v}{\partial t^{2}}=0 \\
(\lambda+\mu) \frac{\partial \theta}{\partial z}+\mu \nabla^{2} w-\rho \frac{\partial^{2} w}{\partial t^{2}}=0 \\
\theta=\frac{\partial u}{\partial x}+\frac{\partial v}{\partial y}+\frac{\partial w}{\partial z} \\
\nabla^{2}=\frac{\partial^{2}}{\partial x^{2}}+\frac{\partial^{2}}{\partial y^{2}}+\frac{\partial^{2}}{\partial z^{2}}
\end{array}\right\}
$$

Where: $u, v$, and $w$ are the displacements in the $x, y$, and $z$ directions respectively; $\mu$ is the Lame constant; $\rho$ is the medium density; $\theta$ is the volumetric strain; $\nabla^{2}$ is the Laplacian operator.

If the medium deformation was caused by wave, only the change in volume without rotation, the equation was

$$
\left.\begin{array}{c}
(\lambda+2 \mu) \nabla^{2} u-\rho \frac{\partial^{2} u}{\partial t^{2}}=0 \\
(\lambda+2 \mu) \nabla^{2} v-\rho \frac{\partial^{2} v}{\partial t^{2}}=0 \\
(\lambda+2 \mu) \nabla^{2} w-\rho \frac{\partial^{2} w}{\partial t^{2}}=0
\end{array}\right\}
$$


The wave by this equation was called P-wave (Qikun, 2009), and the wave equation of the $\mathrm{P}$-wave could be written in the following simple form:

$$
\frac{\partial^{2} \theta}{\partial t^{2}}=v_{p}^{2} \nabla^{2} \theta ; v_{p}=\sqrt{\frac{\lambda+2 \mu}{\rho}}
$$

According to the literature, $(\lambda+2 \mu)$ was called P-wave modulus.

$$
P=\lambda+2 \mu=\rho v_{p}^{2}
$$

In order to obtain the P-wave modulus of the cemented backfill, the density and the P-wave velocity were known. Aiming at the dimension consistency of P-wave modulus and UCS, the UCS prediction formula was established:

$$
\sigma_{c}=a P+b
$$

Where: $\alpha$ was a constant; $b$ was the initial strength

\subsection{UCS prediction formula}

The P-wave modulus of S4 and E3 routes were shown in Figure 7. Origin numerical analysis software was adopted to fit linear of $\mathrm{P}$-wave modulus. Based on $\mathrm{R}^{2}$ coefficient, the fitting coefficients of S4 and E3 routes were $71 \%$ and $75 \%$ respectively, and the fitting effect was better. Therefore, it was proved that the UCS prediction formula was feasible.

$\mathrm{S} 4$ route:

$$
y=0.4+0.00015 x
$$

E3 route:

$$
y=1.2+0.00021 x
$$

Where: $y$ was UCS, MPa; $x$ was the P-wave modulus, MPa.

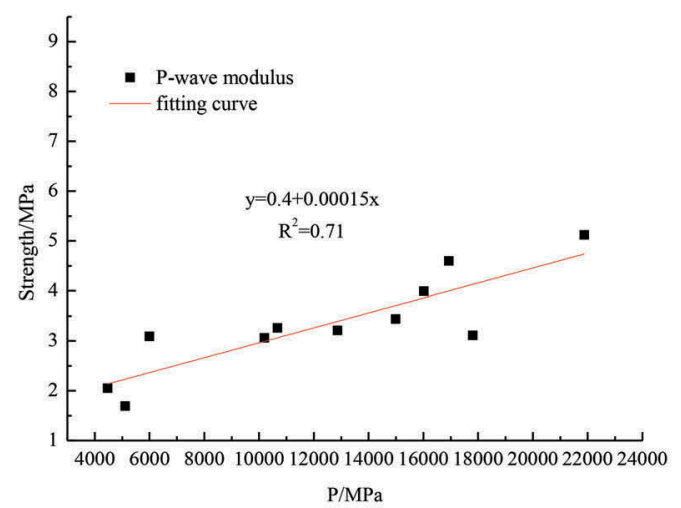

(a) $\mathrm{S} 4$ route

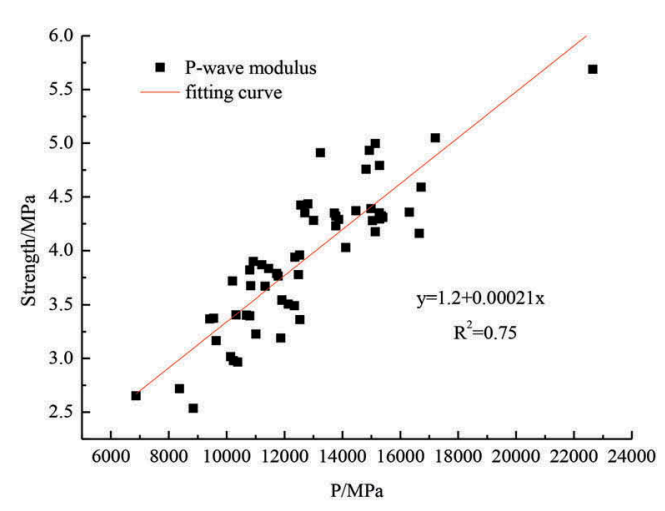

(b) E3 route

Figure 7. The P-wave modulus of S4 and E3 routes. 


\section{CONCLUSION}

(1) The in-situ cemented backfill mass had been evaluated by the comparative analysis of RQD, P-wave velocity and UCS, the filling quality of E3 route was better than the S4 route (without smelting slag), which proved that the gradation of filling aggregate was optimized, and the quality of in-situ cemented backfill was improved by adding smelting slag.

(2) Based on internal links of physicomechanical parameters of in-situ cemented backfill, a relation between UCS and P-wave modulus was established from the perspective of dimensional balance. According to the test data of dry density, P-wave velocity and UCS of cemented backfill, the UCS prediction formula of cemented backfill based on P-wave modulus was obtained by the linear fitting method.

\section{ACKNOWLEDGEMENTS}

This work was supported by the National Key Research and Development Program of China (2017YFE0107000) and the Youth Innovation Fund of BGRIMM (04-2027).

\section{BIBLIOGRAPHY}

Bing, W. and Jun, L. 2003. Analysis of filling process and filling cost of Kalatongke Copper-Nickel Mine. Mining Technology (1), pp. 22-24.

Xiaosu, F. 2009. The application of Gobi aggregate cemented backfill of the underground drift in the Kalatongke mine. Xinjiang Nonferrous Metals (6), pp. 38-39.

Chen et al. 2018 - Chen, Y., Lijie, G., Yaping, Y. 2018. Rheological properties of coarse aggregate paste slurry and calculation of resistance in pipeline transportation. China Mining Magazine 27(12), pp. $178-182$.

Chen et al. 2018 - Chen, Y., Lijie, G., Yaping, Y. 2018. Experimental optimization of filling aggregates in Karatungk Copper-Nickel mine. Nonferrous Metals(Mine Section) 70(6), pp. $38-41$.

Ghirian, A. and Fall, M. 2013. Coupled thermo-hydro-mechanical-chemical behaviour of cemented paste backfill in column experiments. Part I: Physical, hydraulic and thermal processes and characteristics. Engineering Geology (164), pp. 195-207.

Di, W. and Sijing C. 2015. Coupled effect of cement hydration and temperature on hydraulic behavior of cemented tailings backfill. Journal of Central South University (5), PP. 1956-1964.

Weiguo G. and Yulong L. 2007. Brief tutorial of stress wave foundation. Xi'an: Northwestern Polytechnical University Press, 66pp.

Moose et al. 2001 - Moose, D., Zoback M., Bailey L. 2012. Feasibility study of the stability of open hole multilaterals, Cook Inlet, Alaska. SPE Drilling and Completion 16(3), pp.140-145.

Qikun, L. 2009. See world through dimension. Mathematics Communication 33(3), pp. 13-27. 